\title{
LA RELACIÓN ENTRE ESTILOS DE APRENDIZAJE, AUTODIRECCIÓN Y EL DESEMPEÑO ACADÉMICO EN ESTUDIANTES DE MÚSICA DE UNA UNIVERSIDAD MEXICANA
}

\author{
Karmen Arline Herrera Fernández \\ Tecnológico de Monterrey - Universidad Autónoma de Nuevo León \\ a.herrera.fdz@gmail.com \\ Dulce Fátima Camacho Gutiérrez \\ Tecnológico de Monterrey \\ dulce@tecvirtual.mx \\ Yolanda Heredia Escorza \\ Tecnológico de Monterrey \\ yheredia@itesm.mx
}

\begin{abstract}
El objetivo de la presente investigación fue describir la relación entre los estilos de aprendizaje, las habilidades de autodirección y el desempeño académico en un curso de Solfeo. Se utilizó un método cuantitativo, no experimental y transversal-correlacional. La muestra de 76 estudiantes universitarios en Música, respondió el Cuestionario HoneyAlonso de Estilos de Aprendizaje y el Cuestionario de Indagación del perfil autodirigido. Las calificaciones fueron el parámetro del desempeño escolar. Se encontró una correlación moderada e inversa entre el estilo activo y el desempeño en la muestra completa. En el análisis por grupo de estudio se determinaron algunas otras correlaciones.

Palabras clave: Estilos de aprendizaje, autodirección, desempeño, Educación Musical Universitaria, solfeo, CIPA+, CHAEA

The objective of this study was to describe the relationship between learning styles, self-direction skills and academic performance in a course in music theory. A non-experimental and cross-correlational quantitative method was used. The sample of 76 students in a public university Music, answered the Honey-Alonso Learning Styles Questionnaire and the Profile Inquiry Self-Direction Questionnaire. The ratings were school performance parameter. A moderate and inverse correlation between the active style and performance in the whole sample was found. In the analysis by study group, some other correlations were determined.
\end{abstract}

Keywords: learning styles, self-direction, performance, University Music Education, music theory, CIPA +, CHAEA

\section{ANTECEDENTES}

Desde hace varias décadas el estudio del desempeño académico ha sido asunto de interés para diferentes instituciones públicas y privadas. Su investigación a nivel interescolar, local, estatal, nacional e internacional, ha derivado en el descubrimiento de factores asociados que influyen el desempeño escolar, o bien, en precisar de forma específica las cualidades que prevalecen en las escuelas de calidad, entre otros hallazgos.

Dos importantes ejemplos iniciales en esta línea de investigación, son los resultados de Coleman, Campbell, Hobson, McPartland, Mood, Weinfeld y York (1966), quienes presentan importantes conclusiones en temas de segregación racial que cambiaron la política educativa de su tiempo. Edmonds (1979) responde al reporte de Coleman investigando las características presentes en las escuelas de bajos recursos con resultados de alta calidad. En la actualidad, varios organismos internacionales como la OCDE - estudio PISA- (OECD, 2014), la UNESCO -Educación para todos(UNESCO, 2015), y la ONU -Objetivos del Milenio- (UN, 2014), llevan a cabo estudios a gran escala para monitorizar el desempeño y la calidad educativa. Con ello se busca fomentar el desarrollo económico mundial, además del intercambio de ideas entre las naciones y la cooperación para generar condiciones que hagan posible que todos en el mundo puedan acceder a una educación de calidad.

En un contexto universitario en el que la población estudiantil es en su mayoría adulta, los procesos de aprendizaje son guiados por principios andragógicos de los cuales el aprendiz es parte 
central. Bookfield (1986), Cantor (1992) y Knowles (1970) coinciden en destacar la gran importancia de la autodirección para la formación del alumno adulto, ya que posee mayor motivación intrínseca y un alto compromiso interior para aprender y dar respuesta a su necesidad de conocimiento.

Para Rovira (2001: 305) la autodirección es una competencia genérica para la educación universitaria; su utilidad radica en "la disponibilidad para seguir aprendiendo a lo largo de la vida". Así mismo, la Subsecretaría de Educación Media Superior (2008: 15) incluye también el [aprender de forma autónoma] como parte de su listado de competencias genéricas que involucran la habilidad del estudiante para dirigir, organizar y construir su proceso de conocimiento.

Una herramienta de apoyo en el desarrollo de estrategias útiles para mejorar la calidad del estudio, se encuentra en los estilos de aprendizaje, entendidos como la integración entre el estilo cognitivo y las estrategias de aprendizaje (RIDING \& RAYNER, 1999). Gallego y Alonso (2013: 95) refiriéndose a la educación universitaria, sostienen que "muchas de las propuestas y procesos de innovación metodológica, en curso en distintas universidades, encontrarán en la metodología de los estilos de aprendizaje nuevas perspectivas de acción didáctica que faciliten el aprendizaje individual y grupal”.

Gallego y Alonso (2013) insisten en que dentro de las competencias a promover en los niveles universitarios de los estudiantes del siglo XXI, debe hallarse la comprensión del proceso individual de aprendizaje, siendo esta postura una práctica para el autoconocimiento que se reitera en la búsqueda de procesos educativos de más calidad.

Tanto la autodirección como los estilos de aprendizaje son variables implicadas en el aprendizaje adulto. Cantor (1992) clasifica dentro de las características afectivas del aprendizaje adulto: la experiencia vivida, los valores, la autodirección y los estilos de aprendizaje. Estas características, constituyen habilidades, disposiciones, preferencias y tendencias que se manifiestan en el proceso educativo, y por tanto pueden enriquecerlo y tener una implicación en el desempeño académico.

En la Facultad de Música de la universidad mexicana estudiada se preparan profesionales para la ejecución, el canto, la composición, la dirección de coros y la educación musical. Se ofrece un nivel medio superior (llamado Técnico Medio en Música, o Nivel Propedéutico). En este nivel los alumnos que ingresan pueden tener o no estudios musicales previos.

Según Fernández (2003), existen deficiencias en la legislación, el seguimiento y el desarrollo educativo del arte y la música dentro de la educación básica obligatoria, que derivan en una mala formación de habilidades y aptitudes a las que sólo algunas élites tienen acceso. Gutiérrez (2012), atribuye la deserción y bajo desempeño académico en los niveles superiores de la carrera de música, a la incuestionable falta de bases del conocimiento musical desde la educación inicial, y a la escasez de educadores musicales profesionales en primaria y secundaria. De igual manera existen muchas preguntas a resolver sobre los procesos detrás de la evaluación del desempeño en las escuelas superiores de educación musical, debido a la complejidad que encierran los aprendizajes y habilidades musicales esperadas (CHACÓN-SOLIS, 2012).

\section{PLANTEAMIENTO DEL PROBLEMA}

Las distintas teorías de estilos de aprendizaje coinciden en definirlos como las tendencias, preferencias o rasgos cognitivos, fisiológicos y afectivos que influyen en la interacción de un proceso de aprendizaje (KEFEE \& THOMPSON, 1987).

Por su parte la autodirección se refiere al proceso de aprendizaje de un individuo por sí mismo valiéndose o no de ayuda externa, para definir ¿qué?, ¿cómo?, ¿cuándo? y ¿hasta dónde? necesita aprender; estableciendo un plan de acción y autoevaluándose al concluir (KNOWLES, 1975). El perfil de autodirección entonces, agrupa las habilidades que inciden para guiar, ordenar, gestionar y conducir el propio aprendizaje. 
Ya que el desempeño académico sobresaliente de los alumnos en esta disciplina, puede implicar tanto habilidades innatas, como la sensibilidad adquirida a través de la experiencia, el presente estudio busca describir la asociación de factores al desempeño estudiantil. Los estilos de aprendizaje atienden las diferencias individuales de los procesos de aprendizaje del alumnado, y las habilidades de autodirección implican la madurez adquirida por el alumno a través del tiempo para dirigir su aprendizaje.

El curso de solfeo tiene el propósito de desarrollar habilidades indispensables, como lo son: el oído musical, la sensibilidad rítmica, la lectura musical, la entonación de sonidos y la memoria musical. Además de proporcionar conocimientos de teoría de la música y fomentar la escritura, la interpretación y el dictado rítmico y melódico. Con ello el curso de solfeo en el nivel técnico medio, favorece la formación inicial básica del alumno aspirante a ingresar al nivel superior (FAMUS, 2013).

Por lo anterior la pregunta que guió la investigación fue: ¿Qué relación existe entre los estilos de aprendizaje, el perfil de autodirección y el desempeño académico en la materia de solfeo IV de estudiantes de la carrera de Música? De esta pregunta se derivó la pregunta secundaria: ¿Cuáles son los estilos de aprendizaje y el perfil de autodirección de los estudiantes que cursan la materia de Solfeo IV en el nivel propedéutico a la licenciatura en Música?

El objetivo general fue describir si existía relación entre los estilos de aprendizaje, las habilidades de autodirección y el desempeño académico de estudiantes en nivel propedéutico a la licenciatura en Música que cursan la materia de Solfeo IV. El objetivo específico fue describir los estilos de aprendizaje y el perfil de autodirección, presentes en los alumnos del nivel propedéutico en la licenciatura en música que cursan la materia de Solfeo IV.

De manera particular en el contexto de los grupos estudiados, se enfrentan situaciones consecuentes de la ausencia de formación inicial obligatoria dentro de la Educación Básica. En el proceso de formación profesional de la carrera de música los aspirantes parten de una formación musical previa muy elemental o inexistente, y deben desarrollar en un plazo de dos años las habilidades y destrezas que son requeridas para su ingreso al nivel superior. En este escenario, adquiere gran relevancia identificar el impacto de factores que contribuyen a agilizar tanto la asimilación de conocimientos, como el desarrollo de las destrezas musicales por medio del uso de estrategias apropiadas desde etapas iniciales.

El conocer la relación entre estas variables y el desempeño puede proporcionar un marco de referencia para enriquecer la práctica docente, con la inclusión de mecanismos que apoyen la calidad del estudio individual del alumno. Así mismo, el autoconocimiento del proceso de aprendizaje de los alumnos, puede otorgarles nuevas herramientas que apoyen su desarrollo en la disciplina.

En este sentido el presente trabajo exploratorio pretendió contribuir al enriquecimiento del tema, y colaborar con los hallazgos de otras investigaciones en esta misma línea de investigación, pero en otras áreas de estudio.

\section{MARCO TEÓRICO}

El término "desempeño académico" encierra cierta complejidad desde su conceptualización, debido en parte a que existen formas diferentes de referirse a él, algunas de ellas son: "aprovechamiento escolar, aptitud escolar o rendimiento académico" (NAVARRO, 2003: 1). Sin embargo, es posible definir al desempeño académico como la medición cuantitativa de los logros cualitativos -conocimientos, valores, actitudes, o habilidades- alcanzados por un alumno, y que son apropiados a su edad o madurez (GUTIÉRREZ \& MONTAÑEZ, 2012; JIMÉNEZ, 1994)

Sin embargo, Navarro (2003) sugiere que las investigaciones al respecto pueden ser más precisas si se consideran variables asociadas como: la motivación, el auto control y las habilidades sociales por su implicación directa y significativa en el alto o bajo rendimiento académico. 
La evaluación del aprendizaje reflejada por lo general en una nota numérica o calificación, se ha convertido en la forma en que se muestra el desempeño académico de un alumno a lo largo de su formación escolar. Dicha nota ha sido tomada como referente para indicar el éxito o fracaso escolar de una gran cantidad de generaciones de estudiantes (CASCón, 2000). En el caso de esta investigación el desempeño está referido a la forma en que los alumnos logran el desarrollo de las competencias musicales indispensables.

Dentro de la educación musical la evaluación incluye por lo menos tres áreas que integradas generan los aprendizajes musicales. Estas áreas son: los conocimientos necesarios, la ejecución de destrezas y la percepción, -emotividad y sentir del alumno- (CHACóN-Solís, 2013). La observación y diagnóstico de estos aspectos requiere cuidado y dedicación, debido a la complejidad que se encierra en ellos.

González-Pienda (2003) menciona que en el éxito o fracaso escolar intervienen un conjunto de variables conocidas como condicionantes del rendimiento académico. La clasificación realizada por este autor presenta factores personales divididos en variables cognitivas -como la inteligenciaaptitudes, estilos de aprendizaje y conocimientos previos- y variables motivacionales -como autoconcepto, metas de aprendizaje y atribuciones de causa-. Los factores contextuales incluyen variables ambientales -familia, grupo de iguales, ambiente social, clase socioeconómica- variables institucionales -escuela, directivos, ambiente escolar, formación docente etc.- y variables instruccionales -contenidos, métodos de enseñanza, tecnologías, tareas y actividades; y expectativas.

"Estilos cognitivos" es el término usado por la psicología cognitiva para estudiar la forma en que cada persona representa, organiza, procesa, analiza, transforma, utiliza y recuerda la información (CABRERA-AlBerT \& FARIÑAS-LEÓN, 2005; PADILLA, RODRÍGUEZ \& LÓPEZ, 2007). Para Sternberg y Zhang (2000) los estilos se caracterizan por fluctuar entre dos extremos contrastantes (bipolares) y tener un efecto sobre la personalidad y la conducta, sin esto implicar que un estilo sea mejor que otro pues el concepto original no se encuentra ligado al constructo de habilidad o capacidad.

De estas investigaciones los psicólogos educativos han derivado el estudio de las diferentes formas en que las personas realizan actividades de aprendizaje. Keefe y Thompson (1987) definen estilos de aprendizaje como aquellas características cognitivas, fisiológicas y afectivas que se manifiestan en la forma de observar, interactuar o generar una respuesta en un contexto de aprendizaje, y que además muestran ser relativamente estables en el tiempo. El aprendizaje es una actividad compleja que involucra aspectos biológicos, psicológicos y sociales (DíAZ, 2012), por lo tanto el estilo de aprendizaje refleja la forma en que cada individuo se aproxima a él y establece estrategias que le faciliten su alcance.

Honey y Mumford (2000), definieron cuatro estilos de aprendizaje asociados a los ciclos de aprendizaje de Kolb, estos estilos son: Teórico, Reflexivo, Activo y Pragmático. Para cada estilo se distribuyeron características de aprendizaje, actividades favorables, bloqueos presentes y estrategias para la mejora del estudiante. El cuestionario Learning Style Questionarie (LSQ), (HoNEY \& MUMFORD, 2000), fue traducido al español en 1992 por Alonso y Gallego en España, y es conocido como el CHAEA Cuestionario Honey-Alonso de Estilos de Aprendizaje (Alonso, GALLEGo \& HONEY, 2003a). Este instrumento se ha usado mucho dentro de la investigación en idioma español, y sus resultados pueden encontrarse tanto en artículos científicos de investigación para revistas en español y portugués, así como en tesis de posgrado y doctorales.

Algunas de las características del perfil de la persona adulta que aprende, incluyen la autodirección y el aprendizaje con base en la experiencia. Cabe destacar la importancia del papel de la motivación, el autoconcepto, la planificación compartida de contenidos, tareas y metas, el rol activo del alumno, y la presencia de un sentido de mejora mientras se avanza hacia la meta de aprendizaje. (CÁZARES \& PONCE, 2009). 
Según sus autoras, el CIPA Cuestionario de indagación del Perfil Autodirigido (ACEVES \& CÁZARES, 2008), ha probado su efectividad en poblaciones mexicanas, ha cumplido con parámetros que prueban su validez y ha mostrado una mayor estabilidad en la medición del perfil de autodirección. A su vez Cázares y Ponce (2009) señalan que el CIPA logró un mejor ajuste y mostró mayor estabilidad en la medición del perfil de autodirección de la población mexicana, debido a que se integran, como parte del perfil los componentes de la interdependencia y el valor social, aspectos no presentes en otros instrumentos.

Narváez y Prada (2005), estudiaron el aprendizaje autodirigido y el desempeño académico e indagaron sobre aspectos que motivan/desmotivan el aprendizaje. Entre los hallazgos obtenidos se encontró que no existe una correlación entre la autodirección y el desempeño académico y se justificó su causa a la excesiva confianza de los alumnos en los primeros años de su vida universitaria, falta de compromiso o deseo por aprender y responsabilidad por resolver las dificultades del proceso en sí. Los autores concluyen que la capacidad va desarrollándose y que la autodirección toma un tiempo de maduración, es decir evoluciona.

Villa y Heredia (2011) encontraron que existe una relación entre los perfiles de autodirección y el desempeño académico de los grupos analizados. Se obtuvieron valores de correlación positiva lo cual permitió aceptar la hipótesis propuesta, que a mayor perfil de autodirección hay mayor posibilidad de alcanzar un mejor desempeño académico en alumnos de preparatoria.

Por su parte Pintrich y De Groot (1990) se propusieron investigar la relación entre los componentes motivacionales y el aprendizaje autorregulado. Entre los hallazgos obtenidos muestran que existe una correlación entre los altos niveles de auto-efectividad y los altos niveles de uso de estrategias cognitivas. Encontraron que los alumnos que tenían una alta motivación intrínseca eran los que más utilizaban estrategias cognitivas en contraste con los alumnos con baja motivación intrínseca. Las variables motivacionales y cognitivas están relacionadas con el desempeño académico del alumno.

Valle, Cabanach, Rodríguez, Núñez y González (2006) mediante una investigación cuantitativa, investigaron las relaciones entre las metas de desempeño (en sus vertientes de aproximación y evitación) y el uso de estrategias cognitivas y la autorregulación del estudio. Se encontró que además de las metas orientadas al aprendizaje, hay otras metas como las orientadas a la valoración social y a una buena situación laboral futura. Las metas personales son derivadas de una búsqueda de mejora de la autoestima y están relacionadas significativamente con el uso de estrategias cognitivas y de autorregulación del estudio.

Por su parte, Blumen, Rivero y Guerrero (2011) se encontraron con una relación entre el buen rendimiento académico y el estilo teórico en el pregrado, y entre el buen rendimiento académico y el estilo reflexivo y pragmático en el posgrado. Se coincide con otros estudios que destacan un mayor uso de estilos múltiples a medida que los estudiantes se hacen mayores, y una preferencia de los más jóvenes por un estilo particular como es el caso del activo. Al final se descubrió que factores ambientales y de asimilación tienen una relación moderada al desempeño académico, que el interés por el tema (motivación) y una percepción de la capacidad propia para realizar varias actividades a la vez se encuentran presentes en los alumnos con mejor desempeño.

Otros dos estudios con similitudes entre sí, fueron el de Camarero, Martín del Buey y Herrero (2000) y una réplica de este estudio en 2011 por Bahamón, Vianchá, Alarcón y Bohórquez (2013). Ambos utilizaron al CHAEA para medir el estilo de aprendizaje de los estudiantes. En el primero el análisis indica que en el grupo de menor rendimiento académico se encuentran aquellos alumnos que utilizan significativamente el estilo activo, en contraste con los alumnos de mayor desempeño académico que usan el estilo activo de una forma mucho más moderada. Los alumnos de menor desempeño académico tienden a actuar más por intuición y el trabajo metódico y minucioso les requiere mucho esfuerzo, por esta razón las metas a largo o mediano plazo no les resultan atractivas. Por su parte Bahamón et al. (2013) demuestra que la mayoría de los estudiantes 
utiliza una combinación de estilos de aprendizaje. Además, el mismo autor sugiere que los participantes que emplean un estilo de aprendizaje teórico suelen emplear estrategias de aprendizaje de la fase de codificación y obtienen un logro académico medio.

\section{MÉTODO}

El estudio del problema planteó analizar la relación entre una variable dependiente: desempeño académico, que representa las competencias musicales, con las variables independientes: estilos de aprendizaje y perfil de autodirección en una población estudiantil. Debido a que la solución del problema requirió la medición de las variables en un contexto y el análisis estadístico de las mediciones para el establecimiento de conclusiones (HERNÁNDEZ, FERNÁNDEZ \& BAPTISTA, 2010) el enfoque de la investigación fue cuantitativo.

Para efectos del diseño de la investigación se seleccionó un diseño no experimental, ya que no se realizó una manipulación especial o tratamiento deliberado sobre las variables, las cuales fueron registradas en su contexto cotidiano y analizadas posteriormente (HERNÁNDEZ et al, 2010). Así mismo, la investigación fue de corte transeccional o transversal dado que se colectaron los datos en un solo momento y correlacional dado que se buscó determinar una relación entre las variables de estudio (VALENZUEla \& Flores, 2011). Dentro del campo de la enseñanza musical, el presente es un estudio exploratorio en tanto que no se encontraron estudios semejantes que relacionaran estas tres variables.

Se tomó a la población de alumnos que cursaban el último semestre de la materia de Solfeo (IV) del nivel propedéutico de la carrera en música de una universidad pública mexicana. La asignación del muestreo fue por conveniencia y no aleatoria dada las condiciones de tiempo, disponibilidad de espacios, asiduidad de los participantes, localización y facilidades provistas por la institución (VALENZUELA \& FLORES, 2011).

La muestra fue de 76 estudiantes dispuestos en 4 grupos de estudio (equivalente al $80 \%$ de la población total que cursó la materia), cada uno con un profesor diferente. El rango de edad osciló entre los 16 y los 47 años de edad, y se conformó por 30 mujeres (equivalente al 39.47\%) y 46 hombres (equivalente al 60.53\%). Todos ellos aprobaron los primeros tres semestres de sus estudios en la facultad e ingresaron con diferentes niveles de conocimientos previos en el área de música. La finalización de la preparatoria no les es requerida sino hasta iniciar propiamente la licenciatura. El ingreso a la institución puede darse al concluir la educación secundaria o en edades más avanzadas. El nivel propedéutico se distribuye en 4 semestres de instrucción básica previos a la licenciatura, y forma parte de la certificación de Técnico Medio en Música.

Las mayores dificultades de la presente investigación fueron: a) el estudio se realizó solamente en grupos de alumnos del cuarto semestre que concluyeron el curso de solfeo básico IV, b) la falta de una población de mayor tamaño y c) la recopilación de datos y medición de las variables se llevó a cabo solamente en un período del semestre enero-junio de 2015.

Para medir la variable "estilos de aprendizaje" se usó la prueba CHAEA (ALONSO et al, 2003a) la cual es una de las más utilizadas para su medición con cientos de estudios realizados reportando sus calificaciones. El cuestionario consta de una hoja para datos personales y socioacadémicos, instrucciones y 80 ítems que se responden con + o -, según se esté $(+)$ o no (-) de acuerdo con el contenido de cada afirmación. La prueba es distribuida en 20 ítems para cada uno de los estilos (activo, reflexivo, teórico y pragmático), ordenados de forma aleatoria en un solo conjunto. La suma absoluta de la puntuación en cada grupo de 20 items corresponde al nivel en cada estilo (ALONSO, GALLEGO \& HONEY, 1994).

Este estudio se ha practicado con éxito en poblaciones de habla hispana y su alfa de Cronbach para cada estilo de aprendizaje obtuvo grados de aceptabilidad (Activo $=.62$, Reflexivo $=$ .72 , Teórico $=.65$ y Pragmático $=.58$ ). Otros estudios realizados al instrumento han sido las pruebas de análisis de contenidos, análisis de ítems, análisis factorial del total de 80 ítems, análisis factorial 
de cada grupo de 20 ítems para cada factor teórico y análisis factorial de los 4 estilos a partir de las medias totales de sus 20 ítems" (AlONSO et al, 1994: 82)

Para medir la variable de autodirección se utilizó el cuestionario CIPA+ (ACEVES \& CÁZARES, 2008), que ha sido utilizado ampliamente en la población mexicana de estudiantes universitarios, y fue desarrollado en 2002 por la Dra. Yolanda Cázares y ampliado y revisado por la Dra. Nancy Aceves en 2007. Esta prueba es un instrumento de auto-reporte que utiliza una escala de Likert del 1 al 5, en la que 1 indica el grado de mayor afinidad y 5 el de menor afinidad, y está dividido en 5 componentes los cuales son:

- $\quad$ Planificación y selección de estrategias (10 ítems)

- $\quad$ Independencia y autonomía (10 ítems)

- $\quad$ Autorregulación y motivación (14 ítems)

- $\quad$ Uso de la experiencia y la conciencia crítica (9 ítems)

- $\quad$ Interdependencia y valor social (7 ítems)

Los ítems para cada componente se encuentran distribuidos de forma aleatoria en un solo grupo de 50 afirmaciones. La selección del CIPA+ se basó en evidencia que sustenta que el instrumento fue probado en investigaciones en poblaciones de nacionalidad mexicana. Además ha sido validado con técnicas de análisis factorial confirmatorio, y presenta un "índice de confiabilidad Alfa de Cronbach de .956 y un coeficiente de bondad de ajuste del modelo al constructo teórico de .903" (CÁzares \& Ponce, 2009; ACEVEs, LeAl \& PÉrez, 2009: 6).

El procedimiento que se llevó a cabo fue:

- $\quad$ Aplicación del cuestionario CHAEA (cada grupo por separado)

- $\quad$ Aplicación del cuestionario CIPA+ (cada grupo por separado)

- $\quad$ Reunir resultados de cada prueba de forma individual.

- Vaciar resultados y prepararlos para el análisis estadístico.

- Recoger los resultados finales de cada participante de la base de datos Universitaria.

- $\quad$ El análisis estadístico se realizó utilizando el software computacional SPSS.

La estrategia para el análisis de datos fue:

- $\quad$ Se determinó el total por estilo de aprendizaje para cada alumno.

- $\quad$ Se obtiene el valor obtenido en cada componente CIPA+

- Se realizan los cálculos de estadística descriptiva para cada instrumento.

- Se realiza una prueba para determinar la normalidad de la población (SPS-KolmogorovSmirnov)

- $\quad$ Se realizan los cálculos de correlación (SPSS-Rho de Spearman)

- $\quad$ Se hace la triangulación de datos (estadística inferencial Bivariada)

\section{RESULTADOS}

El análisis realizado muestra las medidas de tendencia central (media, mediana, moda y porcentajes), así como las medidas de dispersión (varianza, mínimo, máximo, rango y desviación típica). La Tabla 1 indica el comportamiento de los resultados de la muestra en el CHAEA. 


\begin{tabular}{|c|c|c|c|c|c|c|}
\hline & Total & Activo & Reflexivo & Teórico & Pragmático \\
\hline \multirow[t]{2}{*}{$\mathbf{N}$} & Válidos & 76 & 76 & 76 & 76 & 76 \\
\hline & Perdidos & 0 & 0 & 0 & 0 & 0 \\
\hline \multicolumn{2}{|c|}{ Media } & 52.74 & 11.09 & 15.01 & 13.72 & 12.91 \\
\hline \multicolumn{2}{|c|}{ Mediana } & 54.00 & 11.00 & 16.00 & 14.00 & 13.00 \\
\hline \multicolumn{2}{|c|}{ Moda } & 55 & 14 & 17 & 15 & $15^{\mathrm{a}}$ \\
\hline \multicolumn{2}{|c|}{$\begin{array}{l}\text { Desviación } \\
\text { Típica }\end{array}$} & 6.543 & 3.150 & 2.403 & 2.740 & 2.940 \\
\hline \multicolumn{2}{|c|}{ Varianza } & 42.810 & 9.925 & 5.773 & 7.509 & 8.645 \\
\hline \multicolumn{2}{|c|}{ Rango } & 33 & 13 & 11 & 13 & 13 \\
\hline \multicolumn{2}{|c|}{ Mínimo } & 31 & 4 & 9 & 6 & 5 \\
\hline \multicolumn{2}{|c|}{ Máximo } & 64 & 17 & 20 & 19 & 18 \\
\hline \multicolumn{2}{|c|}{ Suma } & 4008 & 843 & 1141 & 1043 & 981 \\
\hline
\end{tabular}

Tabla 1: Estadística del CHAEA en conjunto y por cada estilo. (a: Existen varias modas, se muestra el menor de los valores)

Los resultados que se presentan en la Tabla 1, indican que la media aritmética con mayor puntuación corresponde al estilo reflexivo con 15.01 y la más baja al estilo activo con 11.09. Los resultados de los participantes de la muestra obtuvieron un promedio más alto para el estilo Teórico y Pragmático, 13.72 y 12.91 respectivamente. Además se ha encontrado que el $19.7 \%$ de la muestra, es decir 15 alumnos, obtuvieron valores similares en dos o más estilos de aprendizaje. Resultados similares se observan en la moda y en la suma total de todos los valores para cada estilo, el estilo reflexivo obtuvo el valor más alto, el estilo activo más bajo, el teórico en segundo lugar y el pragmático en tercero con puntuaciones cercanas.

Los alumnos usan por tanto en forma predominante dos o más estilos de aprendizaje (multimodalidad), o tienen puntuaciones cercanas con 1 ó 2 puntos de diferencia entre los estilos. Alonso, Gallego y Honey (2003b) señalan que todos los discentes tienen cierto nivel de preferencia para cada estilo, el cual evoluciona durante la práctica y el tiempo, por lo que el alumno más capacitado será aquel que pueda aprender bajo cualquier contexto y para ello requieren un buen nivel de preferencia en cada estilo de aprendizaje. Estos valores difieren de lo que se hubiera asumido a priori en tanto que los estudiantes de música tuvieran un estilo de aprendizaje más bien activo ya que el desarrollo de las habilidades musicales requiere de la práctica. Sin embargo, tanto el estilo reflexivo y teórico se pueden ver más asociados a la disciplina requerida para llevar un orden en la práctica musical. Desafortunadamente no fue posible encontrar otros estudios semejantes para contrastar este resultado.

Los alumnos pertenecientes a la muestra cursan el $4^{\circ}$ semestre de estudio, por lo que son estudiantes que han acreditado las materias de los 3 semestres anteriores, y pueden haber diversificado su preferencia de aprendizaje. Esto alude a lo investigado por Blumen, Rivero y Guerrero (2011) que coinciden con otros investigadores al afirmar que los alumnos tienen un mayor uso múltiple de estilos de aprendizaje conforme se van acercando a la edad adulta; y con Bahamón et al, (2013) cuya población de alumnos en nivel superior hace uso principalmente de una combinación de estilos de aprendizaje.

Después de revisar los resultados con base a la suma de los puntos arrojados por el CHAEA, se ha usado el baremo de Honey y Mumford (1986) para interpretarlos con la recodificación por criterio: Muy alto, alto, moderado, bajo y muy bajo. Con este cálculo los valores adquieren un nuevo significado, ya que es posible saber el comportamiento del puntaje obtenido por cada participante en comparación con el rango del grupo completo. A grandes rasgos como lo explica Alonso et al (2003a) las medias obtenidas en cada estilo de aprendizaje de toda la muestra serán 
situadas como una preferencia moderada, y así derivar el cálculo de la preferencia: muy Alta (10\% del puntaje más alto), alta (20\% de quienes han puntuado alto), moderada ( $40 \%$ de quienes han puntuado con nivel medio), bajo (20\% de quienes han puntuado bajo) y muy bajo (10\% de quienes puntuaron más bajo). Este criterio aplicado a la muestra se puede ver en la Tabla 2 a continuación.

\begin{tabular}{|l|l|c|}
\hline Estilo & Criterio & Porcentaje \\
\hline Activo & Muy bajo & $6.60 \%$ \\
\cline { 2 - 3 } & Bajo & $17.10 \%$ \\
\cline { 2 - 3 } & Moderado & $38.20 \%$ \\
\cline { 2 - 3 } & Alto & $23.70 \%$ \\
\cline { 2 - 3 } & Muy alto & $14.50 \%$ \\
\cline { 2 - 3 } & Total & $100.00 \%$ \\
\hline \multirow{5}{*}{ Reflexivo } & Muy bajo & $2.60 \%$ \\
\hline & Bajo & $28.90 \%$ \\
\hline & Moderado & $56.60 \%$ \\
\hline & Alto & $10.50 \%$ \\
\hline & Muy alto & $1.30 \%$ \\
\cline { 2 - 3 } & Total & $100.00 \%$ \\
\hline Muy bajo & $2.60 \%$ \\
\hline & Bajo & $3.90 \%$ \\
\hline & Moderado & $34.20 \%$ \\
\hline & Alto & $55.30 \%$ \\
\hline & Muy alto & $3.90 \%$ \\
\cline { 2 - 3 } & Total & $100.00 \%$ \\
\hline Pragmático & Muy bajo & $5.30 \%$ \\
\hline & Bajo & $19.70 \%$ \\
\hline & Moderado & $27.60 \%$ \\
\hline & Alto & $23.70 \%$ \\
\hline & Muy alto & $23.70 \%$ \\
\cline { 2 - 3 } & Total & $100.00 \%$ \\
\hline
\end{tabular}

Tabla 2: Resultados para estilo activo, reflexivo, teórico y pragmático

Se puede decir entonces que el estilo con mayor cantidad de alumnos en la preferencia es el estilo teórico, con el $55.3 \%$ de la muestra con preferencia Alta. El estilo pragmático obtuvo una preferencia muy alta y alta para un $47.40 \%$ de la población, y el estilo activo suma una preferencia alta y muy alta de $38.2 \%$ igualando a su preferencia moderada (38.2\%). Nótese que el estilo activo, reflexivo y pragmático tiene su mayor porcentaje de frecuencia dentro del criterio moderado, lo que sustenta el uso de estilos múltiples de aprendizaje por el alumnado.

Obsérvese que el estilo reflexivo, después de este análisis, ha obtenido el porcentaje de frecuencia más elevado de la muestra $(56.6 \%)$, pero en la preferencia moderada. Lo cual hace constatar el hecho de que este estilo sea la moda para la muestra total, pero dado que las puntuaciones se acercan mucho a la media se les considera como una preferencia moderada.

Finalmente, al tener en cuenta las mayores puntuaciones para las preferencias: Alta en el estilo teórico y moderado en el estilo reflexivo, es posible describir a grandes rasgos las características de la muestra. Alonso et al (2003b) presenta que los alumnos con estilo teórico son: analíticos, estructurados, metódicos, lógicos, sistemáticos, que cuestionan, que comprenden idea y conceptos complejos, y se presionan intelectualmente. Por su parte los de estilo reflexivo serán quienes: observan, escuchan, piensan antes de actuar, deciden a su propio ritmo, revisan, trabajan sin presión, reúnen información, preparan con tiempo y escuchan diferentes opiniones o puntos de vista. 
En este momento se hizo oportuno revisar la estadística por grupo de estudio, donde las puntuaciones mayores fueron: grupo 1 el $56.3 \%$ calificó en la categoría alto/ muy alto para el estilo pragmático y el $50.1 \%$ es alto /muy alto para el estilo activo, en el grupo 2, el $59.1 \%$ es alto para teórico y el $40.9 \%$ es alto /muy alto en pragmático, en el grupo 3, el $70 \%$ puntea alto/muy alto en el estilo teórico y el $50 \%$ es alto/muy alto en estilo pragmático, y el grupo 4, ubica al $61.1 \%$ de los alumnos en alto /muy alto en estilo teórico y al $44.4 \%$ es alto/muy alto en estilo pragmático.

Al observar lo anterior es notorio que para el grupo 2, 3 y 4, el orden de las preferencias Altas y Muy altas en los estilos, de mayor a menor porcentaje, es el mismo con variantes en las cifras (1.Teórico, 2.Pragmático, 3.Activo y 4.Reflexivo). El grupo 1, coincide en mostrar al estilo reflexivo como el que menos apunta en preferencia Alta, pero la alta preferencia por el estilo Teórico se encuentra detrás del Pragmático y el Activo. Por lo anterior, se puede deducir que tres de los cuatro grupos estudiados presentan una distribución de estilos homogénea, pueden por ello recibir además de la práctica musical experiencial, ideas y conceptos teóricos que complementarán y harán más nutrido su aprendizaje. El conocimiento de las preferencias de aprendizaje del alumnado toma importancia para el profesor, cuando éste es aplicado en la programación de actividades y estrategias para su curso.

La prueba estadística Kuder Richarson o KR20 para medir la confiabilidad de este instrumento dicotómico se presentan en la siguiente tabla (Tabla 3).

\begin{tabular}{|l|c|c|}
\hline \multicolumn{1}{|c|}{ Análisis sobre } & KR-20 & N de elementos \\
\hline CHAEA completo & 0.656 & 80 \\
\hline Estilo Activo & 0.624 & 20 \\
\hline Estilo Reflexivo & 0.430 & 20 \\
\hline Estilo Teórico & 0.550 & 20 \\
\hline Estilo Pragmático & 0.562 & 20 \\
\hline
\end{tabular}

Tabla 3: Índices de fiabilidad KR-20 para el cuestionario CHAEA

Las medidas de tendencia central para la prueba CIPA+ se encuentran en la Tabla (Tabla 4) a continuación. La media de la muestra total es de 90.67 de un total de 250, lo cual indica que la muestra está a penas por encima del nivel óptimo. Si se considera que cuanto más bajo es el puntaje obtenido en el componente mayor desarrollo se tiene de la habilidad, todos los componentes ubican su promedio muy cerca de la mediana y por debajo de la mitad de la escala para cada uno. Esto es un indicador de que los componentes de la muestra están en su mayoría dentro de los niveles óptimo y muy bueno, como se verá más adelante.

\begin{tabular}{|l|l|c|c|c|c|c|c|}
\hline \multicolumn{2}{|l|}{} & CIPA & Comp. 1 & Comp. 2 & Comp. 3 & Comp. 4 & Comp. 5 \\
\hline \multirow{2}{*}{$\mathbf{N}$} & Válidos & 76 & 76 & 76 & 76 & 76 & 76 \\
\cline { 2 - 8 } & Perdidos & 0 & 0 & 0 & 0 & 0 & 0 \\
\hline Media & 90.67 & 19.07 & 20.14 & 24.89 & 15.49 & 11.08 \\
\hline Mediana & 88.00 & 18.50 & 19.00 & 24.00 & 14.50 & 10.00 \\
\hline Moda & $64^{\mathrm{a}}$ & $17^{\mathrm{a}}$ & 18 & $20^{\mathrm{a}}$ & 13 & 7 \\
\hline Desviación Típica & 23.071 & 5.625 & 5.556 & 7.100 & 4.976 & 4.108 \\
\hline Varianza & 532.277 & 31.636 & 30.872 & 50.415 & 24.760 & 16.874 \\
\hline Rango & 133 & 26 & 28 & 40 & 29 & 24 \\
\hline Mínimo & 57 & 10 & 11 & 14 & 9 & 7 \\
\hline Máximo & 190 & 36 & 39 & 54 & 38 & 31 \\
\hline Suma & 6891 & 1449 & 1531 & 1892 & 1177 & 842 \\
\hline
\end{tabular}

Tabla 4: Estadísticas del CIPA+ en conjunto y por cada componente (a: Existen varias modas, se muestra el menor de los valores) 
En la tabla (Tabla 5) se puede observar que en autodirección el 53.9\% de la muestra, ha sido calificado en el nivel "óptimo", y el $40.8 \%$ de la muestra, ha tenido nivel de "muy bueno".

\begin{tabular}{|l|c|}
\hline \multicolumn{1}{|c|}{ Criterio } & Porcentaje \\
\hline Óptimo & $53.9 \%$ \\
\hline Muy bueno & $40.8 \%$ \\
\hline Moderado & $3.9 \%$ \\
\hline Insuficiente & $1.3 \%$ \\
\hline Bajo & $0 \%$ \\
\hline Total & $100.0 \%$ \\
\hline
\end{tabular}

Tabla 5: Resultados del CIPA+ según el criterio calculado para la muestra

Estos datos señalan que los alumnos en su mayoría presentan niveles altos de autodirección. Eso puede deberse, una vez más, a que son estudiantes del $4^{\circ}$ semestre y último del nivel propedéutico, ya que están en vías de iniciar su educación superior en música, por lo que puede asumirse un buen nivel de motivación para continuar con sus estudios. Lo anterior corrobora la investigación de Narváez y Prada (2005), quienes concluyen al analizar una muestra universitaria, que la capacidad de autodirección toma cierto tiempo de maduración y va en evolución a lo largo del estudio profesional. Así mismo, Pintrich y De Groot (1990) encontraron que los alumnos con una alta motivación intrínseca utilizan más estrategias cognitivas en contraste con los alumnos con baja motivación. Alumnos de este último tipo, podrían no formar parte de la muestra analizada, debido a que es posible que hubieran abandonado sus estudios durante los primeros tres semestres de la carrera, o también puede ser que al tener materias no aprobadas estuvieran en un proceso de regularización.

Al realizar el análisis estadístico en cada grupo por separado se encuentra que el grupo 1 cuenta con el $100 \%$ de alumnos cuyos valores obtenidos en el CIPA están en entre los niveles óptimo y muy bueno, el grupo 2 tiene el 90.9\% dentro de estas categorías, mientras que el grupo 3 tiene al $95 \%$ y el grupo 4 al $94.4 \%$. Eso significa que hay una distribución muy similar de alumnos con alto nivel de autodirección en todos los grupos, es decir, se muestra una presencia destacada de cada uno de los componentes que en conjunto integran la autodirección.

Los coeficientes de Alfa Cronbach para la prueba completa y para cada componente son presentados en la tabla (Tabla 6) a continuación.

\begin{tabular}{|l|c|c|}
\hline \multicolumn{1}{|c|}{ Análisis de } & Alfa de Cronbach & N de elementos \\
\hline CIPA+ completo & 0.940 & 50 \\
\hline Componente 1 & 0.824 & 10 \\
\hline Componente 2 & 0.742 & 10 \\
\hline Componente 3 & 0.825 & 14 \\
\hline Componente 4 & 0.819 & 9 \\
\hline Componente 5 & 0.826 & 7 \\
\hline
\end{tabular}

Tabla 6: Coeficientes de fiabilidad Alfa de Cronbach para el CIPA+ y sus componentes

El coeficiente de Alfa Cronbach es de 0.940 para la prueba total, y los índices para el componente $1,3,4$, y 5 presentan valores superiores al 0.819 , en los que solo el componente 2 obtuvo un 0.742. Estos resultados indican que la fiabilidad de este instrumento es aceptable y elevada (HERNÁNDEZ, FERNÁNDEZ \& BAPTISTA, 2010).

Respecto al desempeño, el análisis de frecuencias en la siguiente tabla (Tabla 7), muestra que el $31.58 \%$ de los participantes obtuvieron de 90 a 100, y que 6 alumnos no presentaron o se dieron de baja del curso por lo cual fueron eliminados del estudio. 


\begin{tabular}{|l|c|}
\hline \multicolumn{1}{|c|}{ Calificaciones } & Frecuencia \\
\hline 90 a 100 (Muy bueno) & 24 \\
\hline 80 a 89 (Bueno) & 14 \\
\hline 70 a 79 (Regular) & 19 \\
\hline 60 a 69 (Bajo) & 13 \\
\hline No Presentó & 4 \\
\hline Se dio de Baja & 2 \\
\hline Total & 76 \\
\hline
\end{tabular}

Tabla 7: Porcentajes de calificaciones

En las estadísticas descriptivas para las calificaciones reportadas por los profesores de cada grupo, los resultados se encuentran entre un rango de 40 puntos entre el 100 y el 60. La media de 81.20 se encuentra dentro del intervalo de confianza para la media del $95 \%$, y la moda con más bajo valor fue de 75. Esto indica un desempeño aceptable en general de la muestra.

El procedimiento de bondad de ajuste, para conocer la distribución de la población se utilizó la prueba Kolmogorov-Smirnov sobre cada una de las variables y sobre las calificaciones. En los resultados para cada variable, se encuentra que el grado de significación para todas las variables analizadas es menor a 0.05 , por lo que se advierte que la distribución en todas no es normal (RAZALI \& WAH, 2011). Consecuentemente el análisis de correlación será para una muestra no paramétrica.

\section{Correlación entre variables y desempeño académico}

Para realizar la correlación se tomó la estadística univariada (bivariada). La muestra requirió de estadísticas no paramétricas, y para determinar si existe una relación lineal entre variables a nivel ordinal, se utilizó la correlación $r$ de Spearman o Rho de Spearman (JUÁREZ, VILLATORo \& LÓPEZ, 2002). Se encontró además que la media de cada variable está dentro de los límites del intervalo de confianza para la media al $95 \%$.

La Tabla 8 muestra la correlación entre las calificaciones y el CIPA completo, y para cada uno de sus componentes. En esta se puede observar cómo no existe una correlación lineal entre las variables, Rho Spearman < 0.299 (Juárez, Villatoro y López, 2002). Además el nivel de significancia de relación no cumple con el parámetro $p<o=0.05$, por lo que tampoco se observa significación estadística. Narváez y Prada (2005), han atribuido la falta de correlación entre desempeño y autodirección a otros factores como al compromiso y la madurez del estudiante.

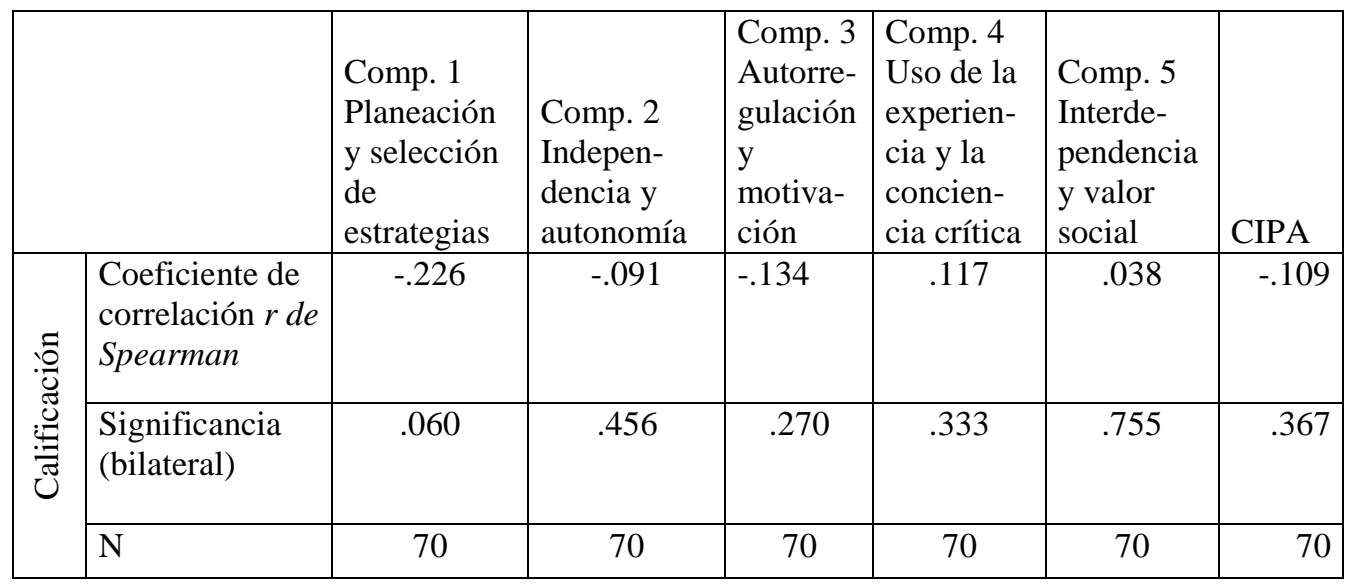

Tabla 8: Correlación Desempeño Académico con CIPA y por componentes

Sobre los estilos de aprendizaje medidos por el CHAEA, y su relación con el desempeño académico, se realizó también la correlación con el coeficiente de Spearman. La siguiente tabla 
(Tabla 9), muestra los resultados de esta prueba de asociación para cada uno de los estilos de aprendizaje y la prueba CHAEA completa. Aquí los resultados, al igual que con el CIPA, no arrojan una asociación lineal, Rho Spearman < 0.299, y solamente el estilo activo muestra un nivel de significancia menor a 0.05 . Esto indica que hay una relación no lineal, estadísticamente significativa e inversamente proporcional entre el estilo activo y el desempeño académico del alumno.

\begin{tabular}{|c|c|c|c|c|c|c|c|c|}
\hline & Activo & Reflexivo & Teórico & Pragmát. & CHAEA & Edad & CALIF. \\
\hline \multirow{3}{*}{ 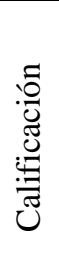 } & $\begin{array}{l}\text { Coeficiente de } \\
\text { correlación } r \text { de } \\
\text { Spearman }\end{array}$ & $-.266^{*}$ & -.041 & .121 & -.147 & -.147 & \begin{tabular}{|l|}
-.030 \\
\end{tabular} & 1.000 \\
\hline & $\begin{array}{l}\text { Significancia } \\
\text { (bilateral) }\end{array}$ & .026 & 736 & .317 & .225 & .223 & .803 & \\
\hline & $\mathrm{N}$ & 70 & 70 & 70 & 70 & 70 & 70 & 70 \\
\hline
\end{tabular}

Tabla 9: Correlación Desempeño Académico con CHAEA y por cada estilo

Esta relación ha sido observada y reportada también por Camarero et al, (2000) y Blumen et al, (2011) en alumnos jóvenes que inician sus estudios y quienes al crecer van utilizando el estilo activo en menor medida o de forma moderada. De igual manera reportan haber encontrado una relación entre el bajo desempeño académico y el estilo activo. Resulta claro que para la mayoría de los alumnos con alto desempeño, el uso del estilo activo debe complementarse con estrategias de aprendizaje que les permitan alcanzar los objetivos académicos de un curso escolar.

Por su parte el grupo 1, como se ve en la Tabla 10, muestra que el componente 1 (Planeación y selección de estrategias), componente 2 (Independencia y autonomía) y el CIPA completo tienen un valor de coeficiente que presenta una relación de magnitud moderada e inversamente proporcional al desempeño académico. El nivel de significancia es de 0.05 para el componente 1 y el CIPA, y de 0.01 para el componente 2. Esto puede indicar que según el criterio con que fue evaluado el grupo a menor presencia de planeación y selección de estrategias, independencia y autonomía; y en el perfil en general se ha obtenido una mayor calificación en desempeño académico o viceversa. Para los otros grupos el uso de estrategias de autodirección fue si no un factor favorable, al menos contribuyó al desempeño en una medida razonable. Lo anterior puede aludir a otros factores no implicados en este estudio que pueden ser de índole variada como la forma de evaluación realizada en cada grupo o la expectativa final de acción entre otros.

\begin{tabular}{|c|c|c|c|c|}
\hline Grupo 1 & & Comp. 1 & Comp. 2 & CIPA \\
\hline \multirow{3}{*}{ 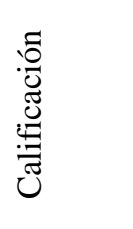 } & $\begin{array}{l}\text { Coeficiente de } \\
\text { correlación (Rho } \\
\text { de Spearman) }\end{array}$ & $-.612^{*}$ & $-.752^{* *}$ & $-.597^{*}$ \\
\hline & Sig. (bilateral) & .020 & .002 & .024 \\
\hline & $\mathrm{N}$ & 14 & 14 & 14 \\
\hline
\end{tabular}

Tabla 10: Relaciones significativas en el análisis por grupos (Grupo 1)

* La correlación es significativa al nivel 0,05 (bilateral).

** La correlación es significativa al nivel 0,01 (bilateral).

Así mismo, Villa y Heredia (2011), en su estudio constatan la existencia de alumnos(as) con bajo desempeño que tienen puntuaciones de moderado a óptimo en el CIPA+ y sus componentes, aunque en menor proporción que en grupos de alumnos con altos promedios. 
Finalmente, en el grupo 3 se encontró un coeficiente de magnitud moderada e inversamente proporcional, con significación estadística del 0.01 entre el estilo activo y el desempeño académico. Además hay una relación de magnitud baja y directamente proporcional con nivel de significancia del 0.05, entre el estilo teórico y el desempeño académico (Tabla 11). Lo anterior sustenta la idea que a mayor presencia del estilo activo, menor puntuación en desempeño académico, mientras que en un nivel de correlación bajo, a mayor presencia del estilo teórico mejor puntuación en desempeño académico.

\begin{tabular}{|c|c|c|c|}
\hline Grupo 3 & & Activo & Teórico \\
\hline \multirow{3}{*}{ 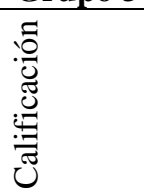 } & $\begin{array}{l}\text { Coeficiente de } \\
\text { correlación (Spearman) }\end{array}$ & $-.630^{* * *}$ & $.463^{*}$ \\
\hline & Significancia (bilateral) & .003 & .040 \\
\hline & $\mathrm{N}$ & 20 & 20 \\
\hline
\end{tabular}

Tabla 11: Relaciones significativas en el análisis por grupo (Grupo 3)

Al respecto, según Alonso, Gallego y Honey (2003b), el sistema educativo tradicional tiende por lo general a premiar al estilo teórico por encima de los otros dadas sus características. Respecto al estilo activo, Camarero, Martín del Buey y Herrero (2000), lo encontraron más presente en alumnos con bajo rendimiento, y Blumen, Rivero y Guerrero (2011), lo encontraron más en alumnos jóvenes que en los alumnos de los grados superiores.

Finalmente, en este sentido y a la luz de los resultados del estudio por grupo de la presente investigación, parece dejarse entre ver que existe una implicación de otros factores como pueden ser la personalidad, estilo de enseñanza y/o de aprendizaje, apreciación, perspectiva, expectativa, criterios y rigor con que se lleva a cabo la evaluación estudiantil. Dada la relativa homogeneidad de los grupos, puede solo suponerse que en los grupos 2 y 4 se haya elaborado una evaluación más uniforme o se hayan seguido estrategias de enseñanza más variadas y diversificadas dirigidas a más estilos. Sin embargo, puede inferirse que tanto los estilos de aprendizaje como el perfil de autodirección son herramientas que proporcionan información significativa tanto para la población estudiantil, como para la planta docente, pero no son del todo un factor que define el rendimiento académico necesariamente para este campo de estudio en particular.

\section{CONCLUSIONES}

En estilos de aprendizaje se encontró que el estilo teórico aparece con una preferencia alta para más de la mitad de la muestra, y que el estilo reflexivo presenta una preferencia moderada también para más de la mitad de los participantes contrariamente a lo que se esperaría a priori de alumnos que estudian música. Existen casos donde los y las estudiantes obtuvieron resultados similares en su preferencia hacia dos o más estilos de aprendizaje (multimodalidad), o mostraron preferencia moderada por más de un estilo de aprendizaje.

En cuanto a la relación de los estilos de aprendizaje y el desempeño académico, se encontró una relación significativa e inversamente proporcional entre el estilo activo y el desempeño académico. Es decir, a mayor presencia del estilo activo menor desempeño académico y viceversa. Este resultado se encontró también al analizar el grupo 3, en el que también se encontró una clara relación inversamente proporcional entre el estilo activo y el desempeño académico y a su vez se presentó una relación directa entre el estilo teórico y el desempeño académico. 
Si se reflexiona sobre el análisis por grupos, se puede pensar que otros factores como el estilo de aprendizaje del profesorado, su estilo de enseñanza, los criterios de evaluación utilizados u otros aspectos diferenciales entre los grupos, pueden adquirir importancia en el desempeño final del alumnado.

Se encontró que el perfil de autodirección de los y las participantes califica para casi la totalidad, en un nivel Óptimo (53.9\%) y Muy bueno (40.8\%). Existe un reducido número de estudiantes que mostraron un nivel de autodirección moderado (3.9\%) e insuficiente (1.3\%). En la muestra estudiada, ninguno de los alumnos obtuvo valores que lo colocaran en el nivel bajo.

Los pocos alumnos con perfil moderado o insuficiente obtuvieron resultados variados en su desempeño; nota regular, alta o baja en el curso escolar.

La cantidad de alumnos con perfil de autodirección óptimo y muy bueno constituyeron para cada caso la mayoría en los grupos de: alumnos aprobados, alumnos no aprobados y alumnos que finalizaron el curso.

No se encontró una correlación con significación estadística entre el perfil de autodirección y el desempeño académico.

Solamente en el análisis por grupos de estudio se ha encontrado que, en uno de ellos a mayor presencia del componente Planeación y selección de estrategias, Independencia y autonomía y del CIPA+ en su conjunto, se tuvo un menor resultado en el desempeño académico. Esta relación inversa es moderada pero con un alto nivel de significancia estadística.

Se ha puesto en claro, dadas las características de la materia de solfeo, la importancia de diversificar el uso de estrategias para alcanzar los objetivos planteados en el curso. Es necesario proponer actividades que favorezcan la práctica musical, la sensibilidad, la motricidad, el razonamiento lógico, la autoobservación y la reflexión, entre otras. El contar con la presencia de diversos estilos de aprendizaje en el aula y la premisa de facilitarles el diversificar sus preferencias, contribuye a que se logre un mejor aprovechamiento y comprensión de los contenidos temáticos planeados. Esto además abre las puertas a la planta docente a generar contenidos que aborden desde actividades experienciales, sensoriales, motrices, teóricas-racionales, de ejecución al instrumento, auditivas o de alguna índole semejante.

Referente al uso de la habilidad de autodirección altamente presente en los y las alumnos(as) participantes, convendría que el profesorado diseñaran actividades o materiales escritos que sirvan para explotar esta habilidad, y coadyuven en la práctica para el desarrollo y perfeccionamiento de las competencias pretendidas en el curso. Un ejemplo de ello puede ser el dedicar tiempo de clase para la explicación de procesos de autoestudio, diversas estrategias de aproximación a los contenidos, realizar prácticas de actividades en forma individual con supervisión y/o sin supervisión, plantear trabajos para realizarse en grupos colaborativos, guiar al alumnado hacia la autoobservación de sus resultados e incluir estrategias de autodescubrimiento y metacognición etc.

Dicho lo anterior, puede resultar provechoso para la institución capacitar a su planta docente para la aplicación de estrategias de autodirección, o en el uso de actividades favorables a los distintos estilos de aprendizaje; y el cómo llevar a cabo un apropiado seguimiento y evaluación de las mismas. En el caso de los estilos de aprendizaje, conviene aplicar una prueba diagnóstico al inicio del período escolar para conocer la distribución de los estilos con mayor y menor presencia en el grupo. Este conocimiento puede ser de ayuda para que el docente logre aplicar los contenidos curriculares de forma variada, de manera que se integren actividades diversas, que favorezcan las 
formas en que cada estilo de aprendizaje se aproxima al conocimiento. Resulta por tanto importante atender conscientemente el estilo de enseñanza del docente.

En este mismo sentido cabe también resaltar, que la naturaleza misma de la clase de solfeo, permite abordar un mismo tema desde diferentes perspectivas. A manera de ejemplo se argumenta que el contenido curricular puede abordarse desde una aproximación teórica (racional), práctica o de ejecución corporal y/o al instrumento (sensorial-motora), sonora o en dictado (auditiva) y en la lectura o escritura (visual). Todas estas aproximaciones favorecen en mayor o menor medida a los estilos de aprendizaje de cada alumno, y al realizarse en conjunto de forma ordenada apoyan al desarrollo, la comprensión y el enriquecimiento del aprendizaje integral en este campo.

\section{REFERENCIAS}

Aceves, N. \& Cázares, Y. (2008). Cuestionario de Indagación del Perfil de Autodirección (CIPA+). Adaptación, Confiabilidad y Validez del Cuestionario de Indagación del Perfil Autodirigido (CIPA) y su evaluación en adultos jóvenes que pertenecen a la sociedad del conocimiento (Tesis doctoral), p. 162. Monterrey: ITESM

Aceves, N., Leal, M. \& Pérez, G. (2009). Efectos de las estrategias didácticas sobre el nivel de autodirección en el aprendizaje en alumnos de Ingeniería. En R. López (Presidencia), X Congreso Nacional de Investigación Educativa-Área 1 Aprendizaje y Desarrollo Humano. Congreso llevado a cabo en Veracruz, México. Consultado en: http://www.comie.org.mx/congreso/memoriaelectronica/v10/pdf/area_tematica_01/ponencias/17 16-F.pdf

Alonso, C., Gallego, D. \& Honey, P. (1994). Los estilos de aprendizaje. Procedimientos de diagnóstico y mejora. Bilbao: Mensajero.

Alonso, C., Gallego, D. \& Honey, P. (2003a). Cuestionario Honey y Alonso de Estilos de Aprendizaje. En: A. Villa (Ed.), Los estilos de aprendizaje. Procedimientos de diagnóstico y mejora (pp. 212-222). Bilbao: Mensajero.

Alonso, C., Gallego, D. \& Honey, P. (2003b). Los estilos de aprendizaje. Procedimientos de diagnóstico y mejora. Bilbao: Mensajero.

Bahamón, M. J., Vianchá, M. A., Alarcón, L. L. \& Bohórquez, C. I. (2013). Estilos y estrategias de aprendizaje, relacionadas con el logro académico en estudiantes universitarios. Pensamiento Psicológico, 11(1), 115-129. Consultado en: http://www.redalyc.org/articulo.oa?id=80127000005

Blumen, S., Rivero, C. \& Guerrero, D. (2011). Estilos de aprendizaje y rendimiento académico en estudiantes universitarios de educación a distancia. Revista de Estilos de Aprendizaje, 4(7), 90105. Consultado en: http://learningstyles.uvu.edu/index.php/jls/article/view/52/27

Bookfield, S. D. (1986). Understanding and facilitating adult learning. San Francisco: Jossey-Bass.

Cabrera-Albert, J. S. \& Fariñas-León, G. (2005). El estudio de los estilos de aprendizaje desde una perspectiva vigostkiana: una aproximación conceptual. Revista Iberoamericana de Educación 37(1), 1-9. Consultado en: http://www.rieoei.org/deloslectores/1090Cabrera.pdf 
Camarero, F., Martín del Buey, F. \& Herrero, F. J. (2000). Estilos y estrategias de aprendizaje en estudiantes universitarios. Psicothema, 12(4), 615-622. Consultado en: http://www.psicothema.com/pdf/380.pdf

Cantor, J. A. (1992). Delivering Instruction to adult learners. Toronto: Wall \& Emerson.

Cascón, I. (2000). Análisis de las calificaciones escolares como criterio de rendimiento académico. Colegio Público Juan García Pérez, España, pp. 1-11. En Red. Consultado en: https://campus.usal.es/ inico/investigacion/jornadas/jornada2/comun/c17.html el 30 de enero de 2015

Cázares, Y. M. \& Ponce, M. P. (2009). La medición de la autodirección; comparación de tres instrumentos OCLI, SDLRS y CIPA, para obtener el perfil de estudiantes en entornos virtuales. En R. López (Presidencia), X Congreso Nacional de Investigación Educativa-Área 7 entornos virtuales de aprendizaje. Congreso llevado a cabo en Veracruz, México. Consultado en: http://www.comie.org.mx/congreso/memoriaelectronica/v10/pdf/area tematica_07/ponencias/04 $\underline{\text { 59-F.pdf }}$

Chacón-Solís, L. A. (2012). ¿Qué significa" evaluar" en música? Revista Electrónica Complutense de Investigación en Educación Musical-RECIEM, 9, 1-25. doi: 10.5209/rev_reci.2012.v9.42805

Coleman, J. S., Campbell, E. Q., Hobson, C. J., McPartland, J., Mood, A. M., Weinfeld, F. D. \& York, R. L. (1966). Equality of Educational Opportunity. Washington: US Government Printing Office.

Díaz, E. (2012). Estilos de aprendizaje. Revista Eidos UTE, (5) 5-11. Consultado en: https://app.ute.edu.ec/Portal/Revista.aspx?idPortal=15\&idCategoria=1144\&idSeccion=1031\&id $\underline{\text { Articulo }=1}$

Edmonds, R. (1979). Effective schools for the urban poor. Educational leadership, 37(1), 15-24. Consultado en: http://www.ascd.org/ASCD/pdf/journals/ed_lead/el_197910_edmonds.pdf

FAMUS (2013). Plan Analítico Solfeo Técnico Medio en Música. Monterrey: Facultad de MúsicaUANL.

Fernández, A. (2003). La educación artística y musical en México. Incompleta, elitista y excluyente. Cuadernos Interamericanos de Investigación en Educación Musical,2(004), 87100. Consultado en: http://www.revistas.unam.mx/index.php/cem/article/view/7322 doi: $10.22201 / 2002.7322$

Gallego, D. \& Alonso, C. (2013). Acción Pedagógica Postdiagnóstico de los estilos de aprendizaje. En J. García, M. Jiménez, T. Martínez \& C. Sánchez (Ed.), Estilos de aprendizaje y otras perspectivas pedagógicas del siglo XXI (pp. 187-200). Texcoco: Colegio de Posgraduados.

González-Pienda, J. A. (2003). El rendimiento escolar. Un análisis de las variables que lo condicionan. Revista Galgo-Portuguesa de Psixología e Educación 8(7), 9-17. Consultado en: http://ruc.udc.es/dspace/bitstream/2183/6952/1/RGP 9-17.pdf

Gutiérrez, S. \& Montañez, G. (2012) Análisis teórico sobre el concepto de rendimiento escolar y la influencia de factores socioculturales. RIDE, (9). Consultado en: http://www.ride.org.mx/docs/publicaciones/09/practica_educativa/Sandra_Gutierrez_OlveraGloria_Silviana_Montanez_Moya.pdf 
Gutiérrez, S. M. (2012). La Música como profesión y disciplina de estudios formales en el México actual. Xipe Totek: Revista trimestral del Departamento Filosofía y Humanidades ITESO, 21(82), 124-141. Consultado en: http://aplicaciones02.fod.ac.cr/igccire/DOCS/Musica_profesion_\%20disciplina.pdf

Hernández, R., Fernández, C. \& Baptista, P. (2010). Metodología de la Investigación. México: McGrow Hill.

Honey, P. \& Munford, A. (1986). The Manual of Learning Styles. Maidenhead: England Ardingly House.

Honey, P. \& Mumford, A. (2000). The learning styles helper's guide. Maidenhead: Peter Honey.

Jiménez, M. (1994). Competencia social: intervención preventiva en la escuela. Infancia y Sociedad: Revista de Estudios, 24, 21-48.

Juárez, F., Villatoro, J. A. \& López, E. K. (2002). Apuntes de Estadística Inferencial. Ciudad de México: Instituto Nacional de Psiquiatría Ramón de la Fuente.

Keefe, J. W. \& Thompson, S. D. (1987). Learning Style: Theory and Practice. Reston: NASSP.

Knowles, M. S. (1970). The modern practice of adult education (Vol. 41). New York: New York Association Press.

Knowles, M. S. (1975). Self-directed learning. New York: Association Press.

Narváez, M. \& Prada, A. (2005). Aprendizaje autodirigido y desempeño académico. Tiempo de educar, 6(11), 115-146. Consultado en: http://www.redalyc.org/articulo.oa?id=31161105

Navarro, R. E. (2003). El rendimiento académico: concepto, investigación y desarrollo. REICERevista Electrónica Iberoamericana sobre Calidad, Eficacia y Cambio en Educación, 1(2), 115. Consultado en: http://www.ice.deusto.es/RINACE/reice/vol1n2/Edel.pdf

OECD. (2014). PISA 2012 results: What students know and can do - Student performance in Mathematics, Reading and Science (Volume I, Revised edition, February 2014). París, Francia: Organisation for Economic Co-Operation and Development (OECD) Publishing. Consultado en: http://www.oecd-

ilibrary.org/docserver/download/9814031e.pdf?expires=1478469271\&id=id\&accname=guest\&c hecksum=8D17C7FB518225962EB347ABC640292E el 18 de septiembre de 2015.

Padilla, V., Rodríguez, M. \& López, E. (2007). Estilos cognitivos y de aprendizaje, en La voz de los investigadores en psicología educativa. México: Ed. Cultura de Veracruz.

Pintrich, P. R. \& De Groot, E. V. (1990). Motivational and self-regulated learning components of classroom academic performance. Journal of educational psychology, 82(1), 33-40. doi: 10.1037/0022-0663.82.1.33

Razali, N. M. \& Wah, Y. B. (2011). Power comparisons of shapiro-wilk, kolmogorov-smirnov, lilliefors and anderson-darling tests. Journal of statistical modeling and analytics, 2(1), 21-33. Consultado en: http://www.de.ufpb.br/ ulisses/disciplinas/normality tests_comparison.pdf

Riding, R. \& Rayner, S. (1999). Cognitive Styles and Learning Strategies. Malwah, NJ: Erlburn Pulishers. 
Rovira, E. (2001). Competencias genéricas en la formación universitaria. Revista de Educación (325), 229-321. Consultado en: https://dialnet.unirioja.es/servlet/articulo?codigo=19417

Subsecretaría de Educación Media Superior. (2008). Competencias Genéricas y el perfil del egresado de la Educación Media Superior. México: Secretaría de Educación Pública. Consultado en: http://ofmx.com.mx/documentos/pdf/Competencias_genericas_perfil_egresado.pdf

Sternberg, R. J. \& Zhang, L. (2000). Perspectives on Thinking, Learning, and Cognitive Styles. Malwah: Erlburn Pulishers.

UN. (2014). Objetivos de desarrollo del milenio: Informe de 2014. Nueva York, E.U.A.: United Nations. Consultado en http://www.un.org/es/millenniumgoals/pdf/mdg-report-2014-spanish.pdf

UNESCO. (2015). Education for all 2000-2015: Achievements and challenges. París, Francia: United Nations Educational, Scientific and Cultural Organization (UNESCO). Consultado en http://unesdoc.unesco.org/images/0023/002322/232205e.pdf

Valenzuela, J. R. \& Flores, M. (2011). Fundamentos de Investigación Educativa Vol. II. Monterrey, México: Digital del Tecnológico de Monterrey.

Valle, A., Cabanach, R. G., Rodríguez, S., Núñez, J. C. \& González, J. A. (2006). Metas académicas, estrategias cognitivas y estrategias de autorregulación del estudio. Psicothema, 18(2), 165-170. Consultado en: http://www.psicothema.com/pdf/3193.pdf

Villa, A. \& Heredia, Y. (2011). Los perfiles de autodirección en alumnos de preparatoria con alto y bajo rendimiento académico. En $\mathrm{H}$. Casanova (Presidencia), XI Congreso Nacional de Investigación Educativa-Área 1 Aprendizaje y Desarrollo Humano, Congreso llevado a cabo en la Ciudad de México, México. http://www.comie.org.mx/congreso/memoriaelectronica/v11/docs/area_01/0515.pdf

Manuscrito recibido el 7/12/2015 y evaluado anónimamente. Aceptado para su publicación el 13/06/2016. 